\title{
A Novel Technique to Perform Microvascular Anastomosis Revisions without Clamps
}

\author{
Amro Harb, BA ${ }^{1}$ Maxwell Levi, BS ${ }^{1}$ \\ Jeffrey Ascherman, $M D^{2}$ \\ ${ }^{1}$ Department of Orthopedic Surgery, Columbia University Irving \\ Medical Center, New York, New York \\ 2 Division of Plastic Surgery, Columbia University Irving Medical \\ Center, New York, New York, United States
}

Rajendra Kumar Kadiyala, MD, PhD ${ }^{1}$

Address for correspondence Jeffrey Ascherman, MD, Division of Plastic Surgery, Department of Surgery, Columbia University Irving Medical Center, Herbert Irving Pavilion, 161 Fort Washington Avenue, Suite: 511, New York, NY 10032 (e-mail: jaa7@cumc.columbia.edu).

J Reconstr Microsurg Open 2018;3:e58-e61.

\begin{abstract}
Background For surgeons learning microsurgery, uneven spacing between sutures while performing microvascular arterial anastomoses is one of the most common technical errors made that can lead to leakage. Based on the previous surgical experience and training of these surgeons, the first option chosen to prevent bleeding is to place a vascular clamp proximal to the anastomosis and an additional suture at the site of the leak. Because this technique may have technical and thrombosis concerns, our study proposes an alternative technique of performing post-anastomotic revisions without the use of clamps.

Methods Our technique involves placing a cotton-tipped applicator under the artery and lifting it to partially occlude flow within the vessel as an additional suture is placed at the leakage site to complete the revision. One-hundred eighty-four microvascular anastomoses were performed on the femoral arteries of 92 Sprague-Dawley rats, and of the 184 anastomoses, 147 had a leak and required a post-anastomotic revision. All revisions were completed using our technique, and no clamps were used during any of the revisions.

Results Of the 147 post-anastomotic revisions completed using our technique, 141

\section{Keywords}

- microsurgery

- anastomosis

- leak

- clamp

- partial occlusion (95.9\%) were patent 2 hours post-revision. The mean operating time for the revisions was 5:03 minutes (range, 1:44-6:30 minutes).

Conclusion Our technique of partially occluding an artery with a cotton-tipped applicator while performing a post-anastomotic revision is a safe and effective alternative to using vascular clamps. Our technique may also reduce technical errors and have a low risk of causing thrombosis when completing post-anastomotic revisions.
\end{abstract}

For surgeons learning microsurgery, one of the most common technical errors made that can lead to leakage after performing microvascular anastomoses on arteries $\sim 1 \mathrm{~mm}$ in diameter is uneven spacing between sutures at the suture line. ${ }^{1}$ At the Microsurgery Training and Research Laboratory of the Columbia University Irving Medical Center, we have noticed that based on the previous surgical experience and training of the surgeons who enroll in our microsurgery training program, the first option chosen to prevent bleeding is to place a vascular clamp proximal to the anastomosis. ${ }^{2}$ An additional suture is then placed at the site of the leak. There are concerns with using this technique, as completely occluding blood flow with a clamp reduces the ability to pinpoint the location of the leak, increases the probability of placing a back-wall stitch due to suture line flattening, and may facilitate platelet accumulation and thrombus formation due to the stagnant flow and increase received

March 23, 2018

accepted after revision

July 25, 2018
DOI https://doi.org/

10.1055/s-0038-1669451. ISSN $2377-0813$.
Copyright $\odot 2018$ by Thieme Medical

Publishers, Inc., 333 Seventh Avenue, New York, NY 10001, USA. Tel: +1(212) 584-4662.
License terms

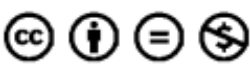


in thrombogenic factors at the suture line. ${ }^{3-5}$ To address these concerns, we propose a simple, novel technique of performing post-anastomotic revisions that uses a cotton-tipped applicator (McKesson Medical-Surgical Inc.; Richmond, VA) to partially occlude blood flow.

\section{Methods}

All procedures described were in compliance with Columbia University's Institutional Animal Care and Use Committee. From September 2017 to February 2018, 46 microsurgeons in training enrolled in our microsurgery training program and performed 184 microanastomoses on the femoral arteries of 92 Sprague-Dawley rats. The mean weight of the rats was $429 \mathrm{~g}$ (range, 343-530 g). The rats were anesthetized with a combination of ketamine $(70 \mathrm{mg} / \mathrm{kg})$ and xylazine $(5 \mathrm{mg} / \mathrm{kg})$. Anesthesia was maintained via an intraperitoneal ketamine bolus. All arteries operated on were $\sim 1 \mathrm{~mm}$ in diameter, and all surgeons used a surgical operating microscope (Zeiss OPMI MD; Carl Zeiss; Jena, Germany) to complete the anastomoses. Each anastomosis was performed using a double clamp with a frame and $10-0$ nylon sutures with a $135^{\circ}$ taper (Surgical Specialties Corporation; Reading, PA). The standard procedure described by Cooley of completing eight interrupted circumferential stitches was followed for each anastomosis. ${ }^{6}$

Of the 184 anastomoses, 147 (79.9\%) had a leak and required a post-anastomotic revision, which was performed using our technique of partially occluding the artery with a cotton-tipped applicator. No clamps were used to perform the revisions, and all revisions were completed with an assistant surgeon holding the cotton-tipped applicator and irrigating with heparinized saline. Patency was verified using the emptyand-refill test, as this test minimizes thrombosis risk. ${ }^{7}$ Our technique was performed using the following guidelines:

1. After completing the anastomosis, apply the fat pad to the artery for 2 minutes for hemostasis. ${ }^{8}$

2. Clean the field with saline and dry with gauze. Apply $1 \%$ lidocaine hydrochloride to the artery for vasodilation. ${ }^{9}$ Examine if there is a leak and confirm that a postanastomotic revision is needed.

3. Place a moist cotton-tipped applicator under the artery and briefly lift it until the flow is completely occluded (-Fig. 1). Irrigate with heparinized saline to clean and gain visualization of the suture line.

4. Once visualization is achieved, gently lower the cottontipped applicator such that the flow within the artery is partially occluded, allowing blood to continue through the artery and leak through the unevenly spaced site (-Fig. 2). Continuous drops of heparinized saline may be applied to pinpoint the location of the leak and identify where the suture needs to be placed. The cotton-tipped applicator may also be lifted and lowered to alternate between complete and partial occlusion, aiding in visualizing where the leak is. In the event that the leak is on the back-wall, it is advised to keep the stay sutures of the 12 and 6 o'clock stitches long to facilitate rotation of the artery on the cotton-tipped applicator.

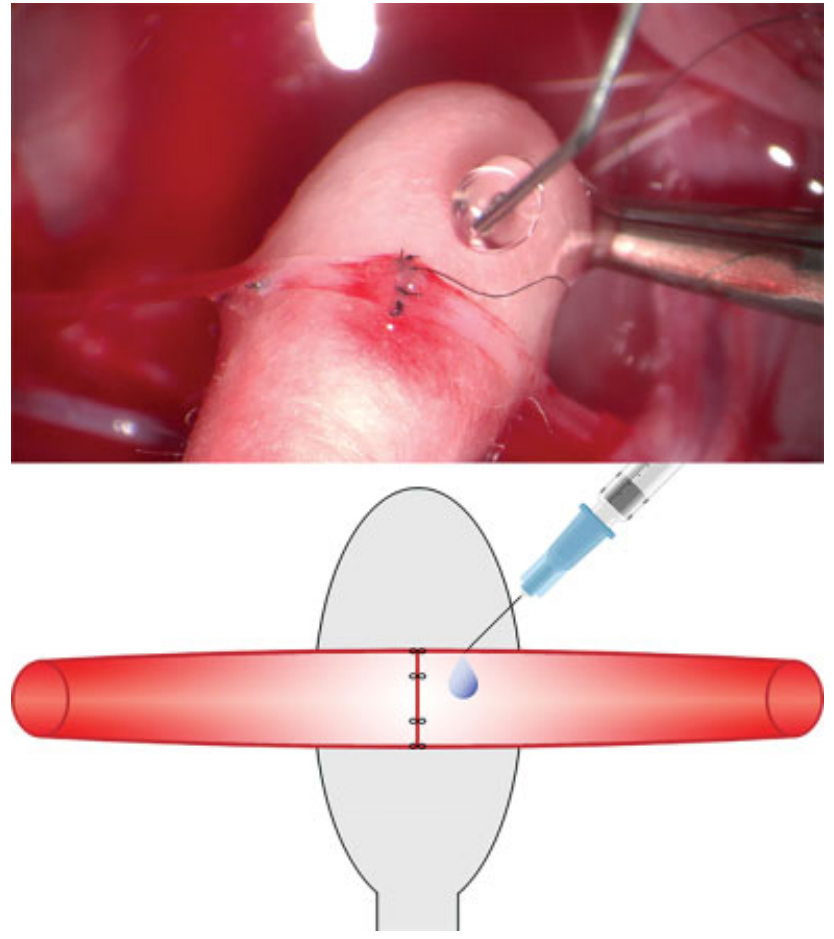

Fig. 1 Complete occlusion. A cotton-tipped applicator is placed under the artery and lifted to completely occlude flow. Heparinized saline is used to clean and gain visualization of the suture line.

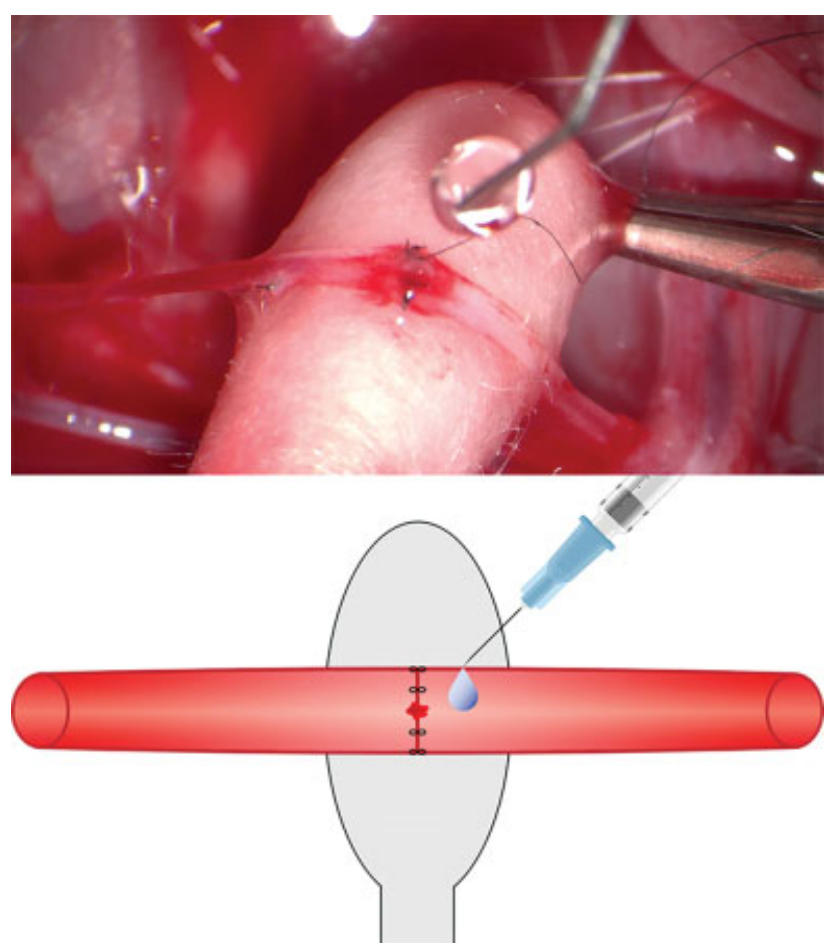

Fig. 2 Partial occlusion. The cotton-tipped applicator is lowered such that the artery is partially occluded and flow is partially restored. Continuous drops of heparinized saline are applied to the suture line to pinpoint the location of the leak. 

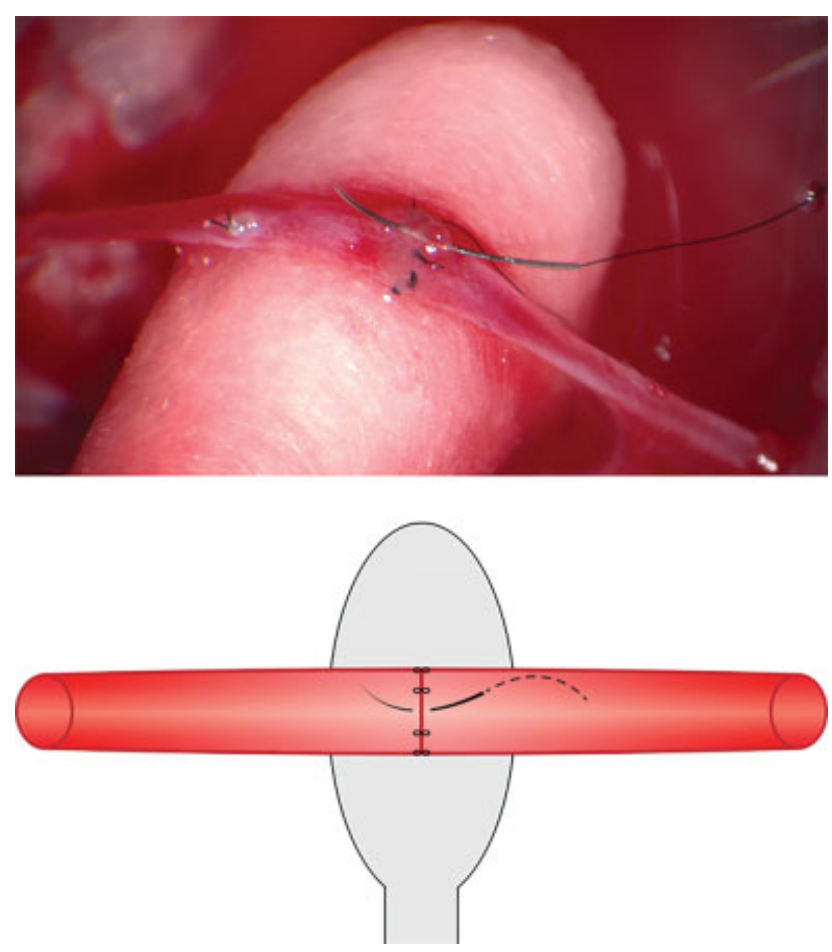

Fig. 3 Partial occlusion. While the artery is still partially occluded, a suture is placed at the site where the leak is visualized.

5. Place a suture at the site where the leak is visualized (-Fig. 3) and lift the tails of the suture to temporarily approximate the arterial ends at the leakage site. If the suture was placed at the correct location, the leak should stop once the arterial ends are approximated. Confirm that no cotton fibers from the cotton-tipped applicator dislodge onto the suture line, as this can lead to thrombus formation. ${ }^{5}$

6. Once the suture is confirmed to be in the correct location and no cotton fibers are present, complete three square knots, remove the cotton-tipped applicator, and examine the artery for additional leaks. Lidocaine may be applied for vasodilation and to expose unseen leaks. ${ }^{9}$ If any leaks are found, the technique may be repeated again.

\section{Results}

Of the 184 microarterial anastomoses, 147 (79.9\%) had a leak and required a post-anastomotic revision. Of the 147 revisions completed using our technique of partially occluding the artery, 141 (95.9\%) were patent 2 hours post-revision. The mean operating time for the revisions was 5:03 minutes (range, 1:44-6:30 minutes).

\section{Discussion}

Our results suggest that partially occluding an artery with a cotton-tipped applicator to complete a post-anastomotic revision is an effective technique, as $95.9 \%$ of the revisions were patent in our study 2 hours post-revision. We tested patency at 2 hours post-revision because previous studies in small animals have shown that thrombosis is most likely to occur in the first 15 to 20 minutes after completing an anastomosis. ${ }^{10}$ The success of our technique may be attributed to it having a low risk of causing thrombosis, as by allowing flow within the artery as the revision is being completed, our technique circumvents the issue of thrombosis occurring due to the conglomeration of stagnant blood and thrombogenic factors at the suture line. ${ }^{3-5}$

Reducing technical errors when completing the revisions may also contribute to why our technique is effective. By allowing flow within the artery as the revision is being completed, our technique allows for the visualization of where the leak is and where the revision needs to be made, which decreases the risk of making unnecessary holes in the artery intima. This improved visualization of the leaks is not possible with clamps, as they completely occlude flow and make it more difficult to pinpoint the location of the leak. Another advantage is that our technique allows for the artery to remain inflated at the suture line as the revision is being completed. This reduces the risk of accidentally placing a back-wall stitch. More specifically, since there is no flow when clamps are placed, the artery remains flat at the suture line, which increases the risk of inadvertently performing a back-wall stitch. Finally, because our technique maintains flow within the artery, it is possible to immediately assess if the revision was successful, as the leak should stop once the suture is placed and the tails are lifted to temporarily approximate the arterial ends at the leakage site. If the leak does not stop, this means the suture was not placed at the correct location, and the surgeon can remove the suture and place it in a better position. This process is less efficient with clamps, as a clamp must first be removed to assess if the revision is successful.

Even though our technique can be performed by one surgeon, we advise having an assistant surgeon to control the cotton-tipped applicator and continuously irrigate the artery with heparinized saline as the revision is being completed. This results in more stable handling of the cotton-tipped applicator, and decreases the risk that it will be lifted too high and cause excess tension on the suture line. An assistant surgeon can also help in verifying that no cotton fibers dislodge onto the suture line, and can take immediate action in removing any cotton fibers if needed. A sponge-tipped applicator may also be used to address the concern of the cotton fibers dislodging onto the suture line. Finally, since partially occluding the artery may lead to turbulent flow, thrombosis can occur if the revision is not completed in a timely manner. ${ }^{11}$ In our study, we found that with an average of 5:03 minutes per revision, 95.9\% of the revisions were patent 2 hours post-revision. An assistant surgeon can help in completing the revisions in this time frame to achieve optimal patency results.

It is important to note that our technique is only effective on small revisions. All arteries operated on in our study were $\sim 1 \mathrm{~mm}$ in diameter. For larger leaks or arteries, a clamp may be necessary to stop the leak and gain visualization of the suture line. Our technique has also only focused on microarterial anastomoses, as the pressure is sufficiently high in arteries to allow blood to continue to flow through the vessel when it is partially occluded. While our technique may be effective for other procedures where the flow is still arterial (such as with interpositional vein grafts), we hypothesize that 
the results would be ambiguous for microvenous anastomoses, and more research needs to be done in this area.

\section{Conclusion}

Our technique of partially occluding arteries with a cottontipped applicator to perform post-anastomotic microvascular revisions is a safe and effective alternative to using vascular clamps.

\section{Funding}

This study was funded by an Orthopedic Scientific Research Foundation grant to the Microsurgery Training and Research Laboratory at the Columbia University Irving Medical Center.

\section{Conflict of Interest}

None.

\section{References}

1 Jose RR, Raja WK, Ibrahim AM, et al. Rapid prototyped sutureless anastomosis device from self-curing silk bio-ink. J Biomed Mater Res B Appl Biomater 2015;103(07):1333-1343
2 Stanley JC, Veith F, Wakefield TW. Current Therapy in Vascular and Endovascular Surgery E-Book. Philadelphia, PA: Elsevier Health Sciences; 2014

3 Rumack CM, Levine D. Diagnostic Ultrasound E-Book. Philadelphia, PA: Elsevier Health Sciences; 2017

4 Wolfe SW, Pederson WC, Hotchkiss RN, et al. Green's Operative Hand Surgery: The Pediatric Hand. Philadelphia, PA: Elsevier Health Sciences; 2010

5 Chang T. Principles, Techniques and Applications in Microsurgery. Singapore: World Scientific; 1986

6 Cooley BC. A Laboratory Manual for Microvascular and Microtubal Surgery. 2nd ed. Reading, PA: Surgical Specialties Corporation; 2009

7 Naides A, Noland R, Lu JG, Akelina Y, Marboe C, Strauch RJ. Histological changes in the rat femoral artery following the use of the empty-and-refill test. J Reconstr Microsurg 2018;34(04): 270-276

8 Economopoulos GC, Sfirakis PD, Zarkalis DA, Loukas LL. Pedicled pericardial fat pad: a useful hemostatic supplement. Ann Thorac Surg 1995;59(03):768-770

9 Kerschner JE, Futran ND. The effect of topical vasodilating agents on microvascular vessel diameter in the rat model. Laryngoscope 1996;106(11):1429-1433

10 Bojrab MJ, Waldron DR, Toombs JP. Current Techniques in Small Animal Surgery. Jackson, WY: Teton NewMedia; 2014

11 Stein PD, Sabbah HN. Measured turbulence and its effect on thrombus formation. Circ Res 1974;35(04):608-614 